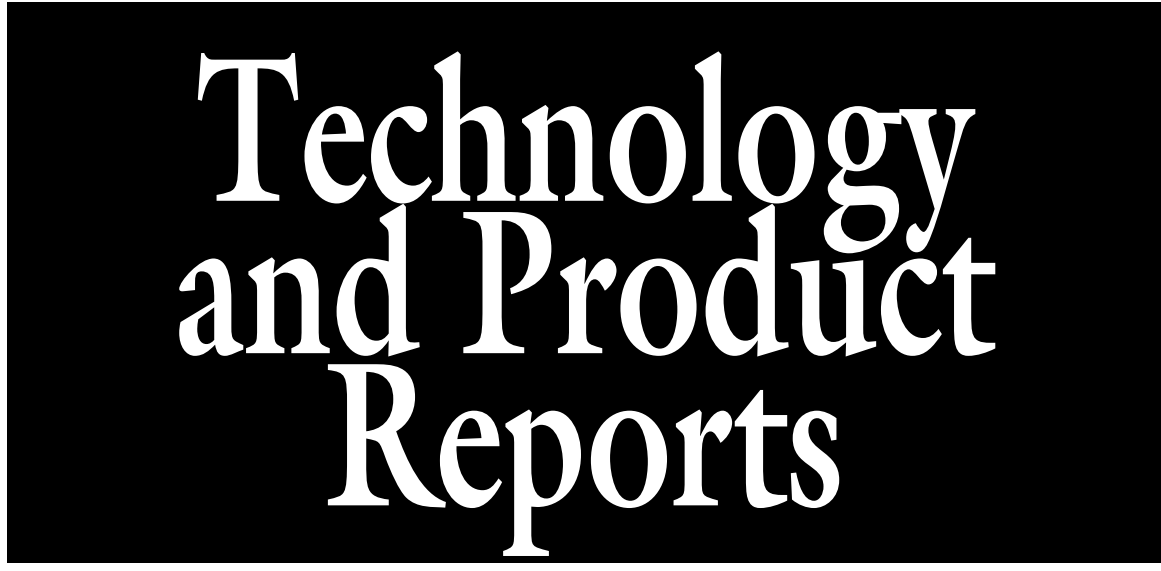

\title{
Proposed Product Label for Electric Lamps Used in the Plant Sciences
}

\author{
Arend-Jan Both ${ }^{1,6}$, Bruce Bugbee ${ }^{2}$, Chieri Kubota ${ }^{3}$, \\ Roberto G. Lopez ${ }^{4}$, Cary Mitchell ${ }^{5}$, Erik S. Runkle ${ }^{4}$, \\ and Claude Wallace ${ }^{1}$
}

AdDitional INDEX WORDs. comparison, efficacy, efficiency, light distribution, lightemitting diode, photon flux, sole-source lighting, supplemental lighting

\begin{abstract}
SUMMARY. Electric lamps are widely used to supplement sunlight (supplemental lighting) and daylength extension (photoperiodic lighting) for the production of horticultural crops in greenhouses and controlled environments. Recent advances in light-emitting diode (LED) technology now provide the horticultural industry with multiple lighting options. However, growers are unable to compare technologies and LED options because of insufficient data on lamp performance metrics. Here, we propose a standardized product label that facilitates the comparison of lamps across manufacturers. This label includes the photosynthetically active radiation $(P A R)$ efficacy, $P A R$ conversion efficiency, photon flux density output in key wave bands, as well as the phytochrome photostationary state (PSS), red/far red ratio, and graphs of the normalized photon flux density across the 300-900 $\mathrm{nm}$ wave band and a horizontal distribution of the light output.
\end{abstract}

S pecialty crop growers have typically used high-pressure sodium lamps for supplemental lighting and incandescent and compact fluorescent lamps for photoperiodic

Partial funding for this work was received from the National Institute of Food and Agriculture Specialty Crop Research Initiative grant 2010-51181-21369.

${ }^{1}$ Department of Environmental Sciences, Rutgers University, 14 College Farm Road, New Brunswick, NJ 08901

${ }^{2}$ Department of Plants, Soils and Climate, UT State University, 4820 Old Main Hill, Logan, UT 84322

${ }^{3}$ School of Plant Sciences, University of Arizona, 1140 E. South Campus Drive, Tucson, AZ 85721

${ }^{4}$ Department of Horticulture, Michigan State University, 1066 Bogue Street, East Lansing, MI 48824

${ }^{5}$ Department of Horticulture and Landscape Architecture, Purdue University, 625 Agriculture Mall Drive, West Lafayette, IN 47907

${ }^{6}$ Corresponding author. E-mail: both@aesop.rutgers. edu.

doi: 10.21273/HORTTECH03648-16 lighting in greenhouses. In plant propagation facilities for tissue culture and research, sole-source electric lighting has typically been used, with fluorescent tubes as the main light source that is occasionally supplemented with incandescent lamps. Supplemental lighting typically requires high intensities (at least 50-100 $\mu \mathrm{mol} \cdot \mathrm{m}^{-2} \cdot \mathrm{s}^{-1}$ ) because it is intended to increase photosynthesis and thus plant growth, whereas photoperiodic lighting only requires a low intensity $\left(1-3 \mu \mathrm{mol} \cdot \mathrm{m}^{-2} \cdot \mathrm{s}^{-1}\right)$ to regulate flowering of photoperiodic crops.

As LED technology developed and became less expensive, LED lamps have been used for a wide range of lighting applications, including for horticultural crop production. LED lamps have several advantages over traditional lamp types: l) LEDs can be manufactured to emit a variety of narrow wave band colors, which facilitates generating a specific (sometimes adjustable) spectral output, 2) LEDs produce less radiant heat per photon, 3) LEDs can have a longer operating life, 4) individual LEDs are low power and small in size, allowing for small, low-wattage lamps (arrays) that can be placed closer to the plants, and 5) LEDs can be designed to produce light with a narrowly focused beam angle, potentially increasing the light-use efficiency by maximizing the capture of light by plant surfaces.

When prices started to drop and LED intensity and reliability increased through improved technologies and manufacturing processes, several companies started supplying LED lamps for plant growth applications. Despite a few early adopters, many growers were unsure of the financial benefits of these new lighting systems. In addition, the novelty of LED technology, the myriad possibilities for operational strategies, the lack of research data, and the often significantly higher investment costs caused trepidation among growers. Although many plant scientists are conducting research on best practices for LED applications (e.g., Mitchell et al., 2015) and some of the key performance metrics for horticultural lighting have been characterized (e.g., Nelson and Bugbee, 2014), so far little has been done to standardize and report the primary performance metrics of LEDs that are important for plant growth applications.

\begin{tabular}{llll}
\hline $\begin{array}{l}\text { Units } \\
\text { To convert U.S. to SI, } \\
\text { multiply by }\end{array}$ & U.S. unit & SI unit & $\begin{array}{l}\text { To convert SI to U.S., } \\
\text { multiply by }\end{array}$ \\
\hline 0.3048 & $\mathrm{ft}$ & $\mathrm{m}$ & 3.2808 \\
25.4 & inch $(\mathrm{es})$ & $\mathrm{mm}$ & 0.0394 \\
3.6000 & $\mathrm{kWh}$ & $\mathrm{MJ}$ & 0.2778 \\
$\left({ }^{\circ} \mathrm{F}-32\right) \div 1.8$ & ${ }^{\circ} \mathrm{F}$ & ${ }^{\circ} \mathrm{C}$ & $\left({ }^{\circ} \mathrm{C} \times 1.8\right)+32$ \\
$\left({ }^{\circ} \mathrm{F} \div 1.8\right)+255.37$ & ${ }^{\circ} \mathrm{F}$ & $\mathrm{K}$ & $(\mathrm{K}-255.37) \times 1.8$
\end{tabular}




\section{Available evaluation tools}

These challenges are not unique to the lighting systems used for plant growth and development. Performance metrics exist for residential and commercial applications (human vision) that allow for a basic comparison among the large number of lamps and lighting systems that are available. For example, the U.S. Department of Energy has developed a Lighting Facts $®$ label that most manufacturers now incorporate on their packaging. These labels (Fig. 1) include the following information: power consumption (watts), light output across the visible spectrum (lumens), efficacy (lumens/watt), color rendering index (CRI), and correlated color temperature (CCT). Some of these labels also include information about expected lamp life based on a specific number of daily operating hours, and yearly energy cost based on daily operating hours and electricity cost (dollars/kilowatt-hour). In some cases, these labels also include information about lumen maintenance (the expected drop in light output after a certain number of operating hours compared with that of a new lamp), manufacturer warranty on the lamp, and the inclusion of any

\section{lighting facts}



Fig. 1. Example of a Lighting Facts ${ }^{\circledR}$ label (U.S. Department of Energy) that includes information about operating characteristics for an LED lamp used for visible lighting applications. This label is not suitable for plant lighting applications; (K $255.37) \times 1.8={ }^{\circ} \mathrm{F}$. hazardous chemicals (e.g., mercury). Consumers can use this information to make informed purchasing decisions. However, no such label is available for lamps that are used, specifically designed, or both for plant growth applications.

\section{Proposed product label for lighting applications in the plant sciences}

Here, we propose a product label for all lamps intended for plant growth applications (Fig. 2). The information shown in the figure does not imply an endorsement of one particular lamp over others available from different manufacturers; the figure is strictly intended as an example. The following paragraphs describe the content of the text and figure boxes on the label.

EleCtRICITY AND VISIBLE LIGHT CHARACTERISTICS. Like residential and commercial users of lamps designed for human applications, growers are interested in input voltage, current draw, and power consumption. Because it is important for horticultural lamps to provide light to help humans distinguish subtle color differences associated with plant nutrient deficiencies, diseases, tiny insects, fruit color, and other types of plant stress, it is useful to include metrics that are of interest for human applications (lumen output, CRI, and CCT), although these metrics are not directly useful for applications in the plant sciences. The highest CRI value is 100 (daylight), white LEDs typically have values exceeding 90, and high-pressure sodium lamps have a CRI of 24, whereas some lamps emit light with a negative CRI value. The CCT represents the color appearance of light emitted by a lamp measured in degrees Kelvin. Light emitted from lamps with a CCT below $3200 \mathrm{~K}$ is considered warm (more red in color), whereas light with a CCT above $4000 \mathrm{~K}$ is considered cool (more blue in color) in appearance. Color temperature can be confusing because low temperatures are associated with warmer colors. This is due to Wein's Law, which states that the maximum wavelength of radiation is inversely proportional to the surface temperature of the radiation source in Kelvin.

WaVE BANDS. The portion of solar radiation defined as $P A R$
(400-700 $\mathrm{nm}$ ) is slightly different compared with the portion of the solar spectrum associated with human vision [380-780 nm (Fig. 3)]. In addition, a portion of ultraviolet [UV (300-399 nm) including a portion of UV-B $(280-315 \mathrm{~nm})$ and all of UV-A (315-399 nm)] and far red (sometimes defined as 700-799 $\mathrm{nm}$ ) radiation can impact plant growth and development, as can infrared [IR (heat)] radiation $(800 \mathrm{~nm}-$ $1 \mathrm{~mm})$. The latter is more difficult to quantify and primarily influences plant temperature (for details see Nelson and Bugbee, 2015) and is therefore not included in the proposed label. The proposed label includes a table that quantifies the photon flux density generated across distinctive wave bands associated with ultraviolet (300-399 nm), blue (400$499 \mathrm{~nm})$, green $(500-599 \mathrm{~nm})$, red (600-699 nm), far red (700-799 $\mathrm{nm}$ ), and (a portion of) near IR $(800-900 \mathrm{~nm})$ radiation. These data were collected from a measurement at a distance of $2 \mathrm{ft}$ directly below the center of the lamp. If a lamp is designed with a nonuniform placement distribution of differently colored LEDs, resulting in changing spectral output depending on the measurement location, we recommend this be noted on the label.

Spectral Intensity. The sensitivity of plants to different wavelengths of light is significantly different from the sensitivity of the human eye (Fig. 3). Absorption of light by plants is accomplished with multiple photosynthetic pigments, and multiple photoreceptors are used to alter plant morphology. So, it is important to provide detailed information about the spectral output of lamps used for plant growth applications. Because the lamp output depends on power input, the normalized (i.e., all measured values are divided by the largest value) photon flux density is shown to facilitate comparison of low- and high-wattage lamps. We propose that measurements be made and reported in wavelength intervals no larger than $3 \mathrm{~nm}$.

Light Distribution. For most commercial plant growth applications, lamp installations are designed to provide a uniform photon flux density over a specific growing area. Lamps are typically mounted at a fixed height (or distance) above (from) the 


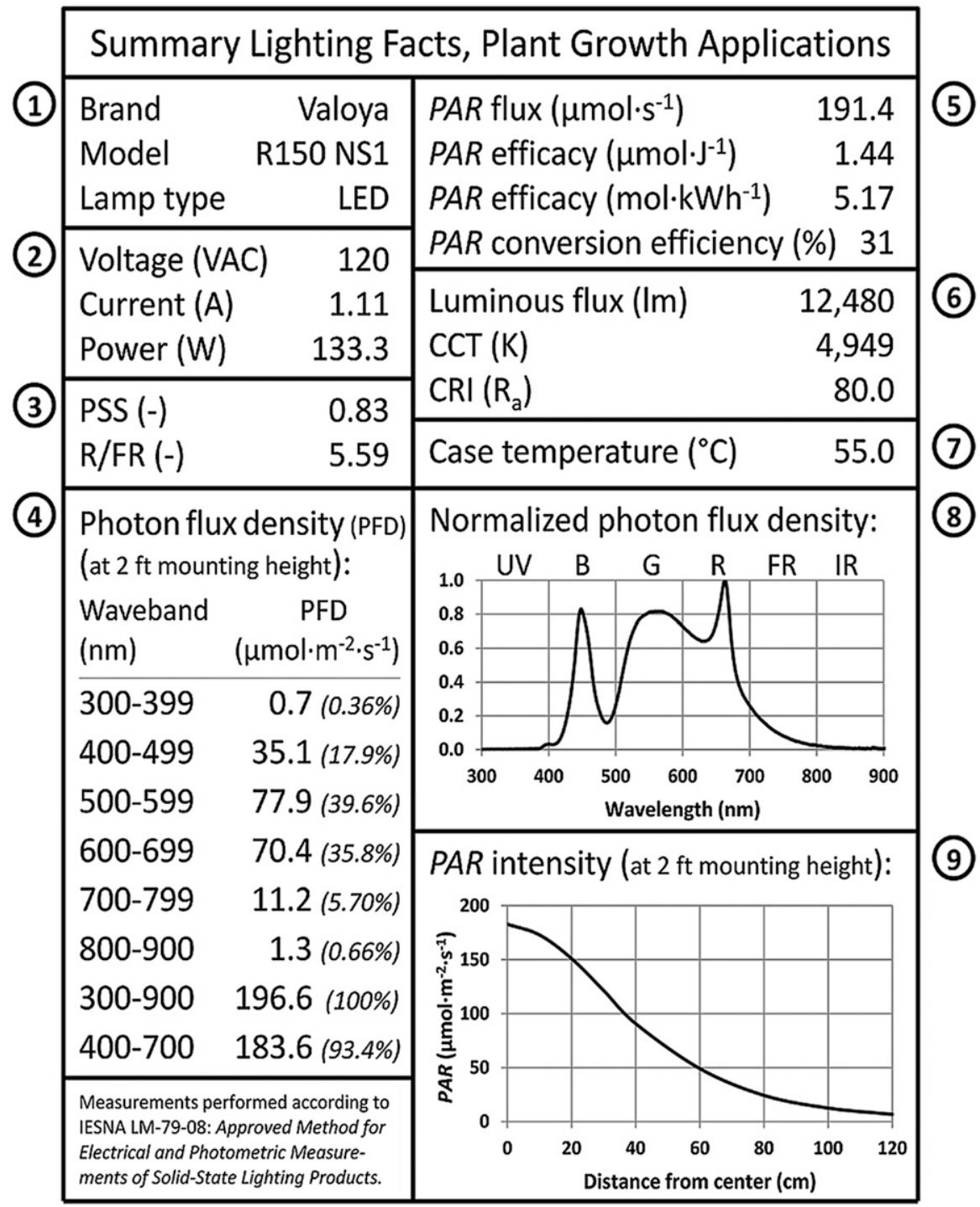

Fig. 2. Example of the proposed product label for a light-emitting diode (LED) lamp specifically designed for plant growth applications. PSS is the phytochrome photostationary state, using values from Sager et al. (1988), which is a dimensionless ratio indicated by $(-)$. $R / F R$ is the red/far red ratio and is calculated from the sum of the photons between 640 and 680 (red) divided by the sum of the photons between 710 and $750 \mathrm{~nm}$ (far red); VAC = voltage in an alternating current circuit, $\mathrm{A}=$ ampere; $P A R=$ photosynthetically active radiation; $\mathrm{CCT}=$ correlated color temperature; $\mathrm{CRI}=$ color rendering index; $\mathbf{R}_{\mathrm{a}}=$ international standard color rendering index as defined by the Commission Internationale de l'Eclairage; UV = ultraviolet; $\mathrm{B}=$ blue; $\mathbf{G}$ = green; $R=$ red; $F R$ = far red; $I R$ = infrared. Legend (numbers to the left and right of the label): (1) lamp information, (2) electrical operating characteristics, (3) commonly used numbers that predict certain spectral effects, (4) photon flux output across commonly used wave bands, including the $P A R$ waveband, (5) lamp output and efficiency numbers, (6) lamp output characteristics used for human vision applications, (7) temperature of the LED array measured at a representative location during testing, (8) normalized spectral output across the 300-900 nm waveband, and (9) $P A R$ intensity below the lamp installed at a fixed mounting height of $2 \mathrm{ft}$ [ $0.6 \mathrm{~m}$ (for this particular lamp, the $P A R$ distribution pattern was independent of the direction in which the sensor was moved away from the center below the lamp $)] ;(\mathrm{K}-255.37) \times 1.8={ }^{\circ} \mathrm{F},\left(1.8 \times{ }^{\circ} \mathrm{C}\right)+32={ }^{\circ} \mathrm{F}, 1 \mathrm{kWh}=3.6000 \mathrm{MJ}$.

crop. It is, therefore, important to know the light distribution so that the uniformity can be evaluated. The proposed label includes a graph that shows, for a fixed mounting height of $2 \mathrm{ft}$, the average change in $P A R$ intensity as a sensor is moved away (in a horizontal plane) from a point directly below the center of the lamp. For the particular lamp described on the proposed label (Fig. 2), there was little difference in the change in $P A R$ intensity relative to the direction in which the observer moved away from the center (data not shown), and hence, the changes were averaged into a single curve as shown.

SPECTRAL EFFECTS ON PHYTOCHROME: RED/FAR RED RATIO AND PHYTOCHROME PHOTOSTATIONARY STATE. Phytochrome is a photoreceptor that controls many morphological responses in plants. It is primarily sensitive to the red and far red radiation, but it is also sensitive to blue radiation. Two spectral ratios have been used to predict spectral effects on this photoreceptor: the red/far red ratio and the PSS (sometimes referred to as PPS). The red/far red ratio does not have a definitive definition, but we recommend adoption of the ratio of the sum of the red photons (for this purpose defined as 640-680 $\mathrm{nm}$ ) to the sum of the far red photons (for this purpose defined as $710-750 \mathrm{~nm}$ ) because these wavelength ranges encompass the spectral absorption of each form of this photoreceptor. The PSS is defined as the ratio of the relative proportion of the far red absorbing state of phytochrome to the total amount of phytochrome at equilibrium and was calculated according to the method first described by Gardner and Graceffo (1982) that was later modified by Sager et al. (1988). The calculated PSS value for midday sunlight is 0.7 , but can decrease to 0.1 for shaded conditions under a closed plant canopy. Generally, extension growth (shade avoidance response) increases as the PSS value decreases (as the red/far red ratio decreases).

Temperature. The light output of all lamps, including LED lamps, is influenced by the operating temperature. An example is shown in Fig. 4. Recommended testing procedures (IESNA, 2004, 2008a) specify that the ambient temperature should be maintained at $25^{\circ} \mathrm{C}\left( \pm 1^{\circ} \mathrm{C}\right.$ for LED lamps and $\pm 5{ }^{\circ} \mathrm{C}$ for high-intensity discharge lamps). This temperature should be measured close to the lamp $(1-1.5 \mathrm{~m})$, and the sensor should be shielded from any direct radiation from the light source. We propose 
that any deviations from the target ambient temperature are reported on the label either as 1) a different ambient temperature that was reached after stabilization (usually after 30120 min of lamp operation) or 2) the stabilized case temperature (as defined for LED lamps only). The case temperature is defined as the temperature at a representative location on the solid surface to which the LEDs are attached (usually opposite the side to which the heat sink is attached). Typically, the lamp manufacturer provides information about the proper location for this measurement.

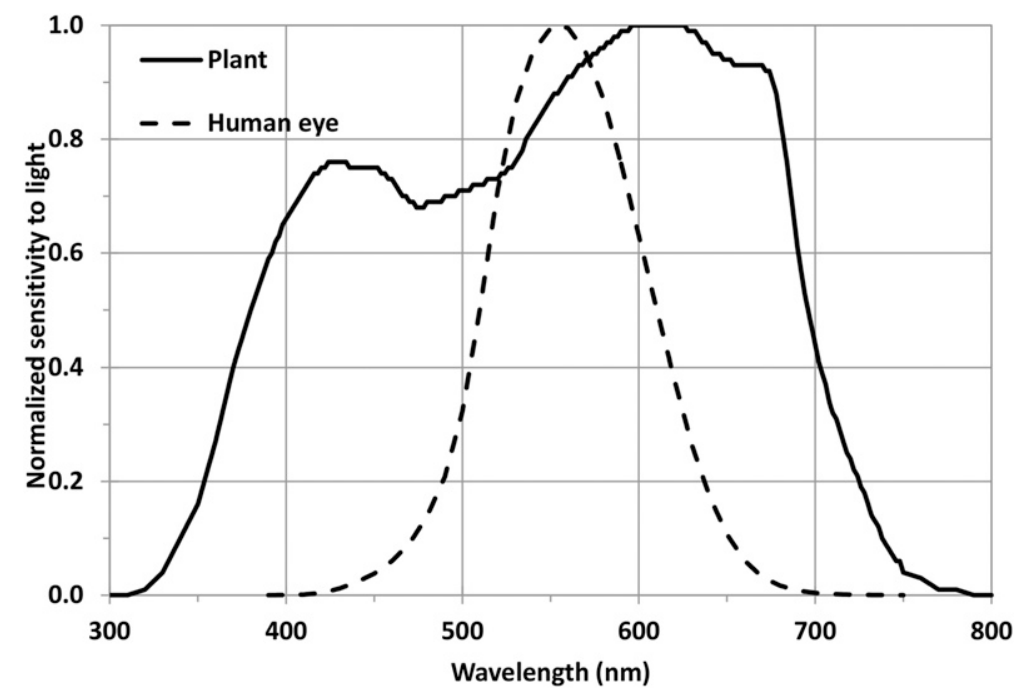

Fig. 3. Comparison of the (photopic) light sensitivity of the human eye (Commission Internationale de l'Eclairage, 1931) vs. the instantaneous photosynthetic efficacy (photon weighted) of radiation reaching plant leaves (McCree, 1972; Sager et al., 1988). The curve for photosynthetic efficacy was developed from short-term measurements on single leaves, in low light and is therefore not a definitive standard for photosynthesis in whole plants.

Photosynthetically active radiation has historically been defined as the photon flux with equal weighting of photons between 400 and $700 \mathrm{~nm}$. Note the (sometimes significant) differences in sensitivity between plants and the human eye with respect to wavelength.

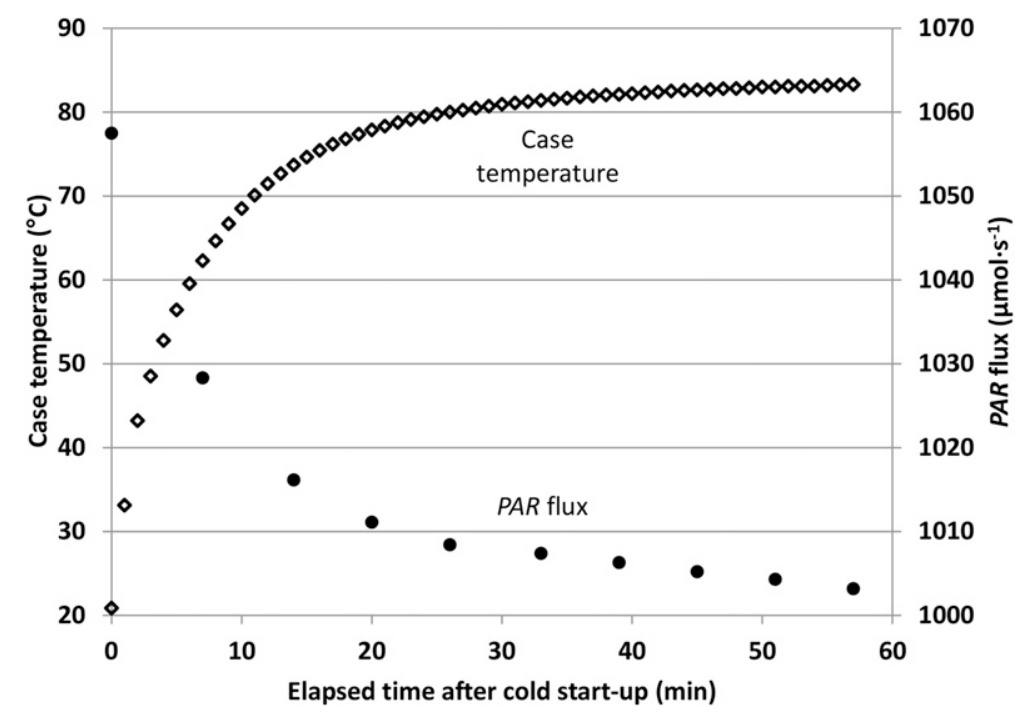

Fig. 4. Example of the potential impact of the case temperature (open diamonds) on the photosynthetically active radiation $(P A R)$ flux $[400-700 \mathrm{~nm}$ (solid circles)] output of a light-emitting diode lamp. During the time period shown, the case temperature increased by $300 \%$, whereas the $P A R$ flux dropped by $5 \%$; $\left(1.8 \times{ }^{\circ} \mathrm{C}\right)+32={ }^{\circ} \mathrm{F}$.

Reporting the case temperature removes any ambiguity about the temperature conditions during the test procedure.

Heat generation. Especially for controlled-environment applications, it can be important to know how much heat is generated by a particular light source. The amount of heat generated by lamps can be a benefit when supplemental heating is needed to maintain a desired temperature set point, or it can be a burden when it results in excess heat that needs to be removed by a cooling system. To (indirectly) determine the heat generation by a lamp, the $P A R$ conversion efficiency is included on the label. The PAR conversion efficiency was calculated as the ratio of the radiant energy output (watts) across the $P A R$ wave band divided by the total electric energy consumption of the lamp (watts). Using the $P A R$ conversion efficiency value, the amount of heat generation can be determined. For example, the LED lamp used for Fig. 2 has a $P A R$ conversion efficiency value of 0.31 , meaning that of the electric energy consumed by the lamp $\left(\mathrm{W}_{\mathrm{e}}\right), 31 \%$ is emitted as radiant energy $\left(\mathrm{W}_{\mathrm{r}}\right)$ in the $P A R$ wave band and the remaining $69 \%$ outside the $P A R$ wave band and mostly converted to heat.

\section{Discussion}

MEASUREMENT EQUiPMENT FOR aCCURATE MEASUREMENTs. Lightintensity readings can be measured with a PAR sensor that has been spectrally calibrated to a spectroradiometer for the individual lamp, but because lamp types produce light with different spectral compositions, it is recommended that all intensity measurements be performed with a cosine-corrected and calibrated spectroradiometer, using a wavelength resolution of no more than $3 \mathrm{~nm}$.

Lamp efficacy measurements are typically performed using an integrating sphere or a goniophotometer. These devices measure either uniformly scattered light (integrating sphere) or light emitted at a range of different angles (goniophotometer). These measurements can then be used to determine the total (i.e., emitted in all directions) light output. If necessary, the measured radiant power appropriate for visible applications 
(in watts/nanometer) can be converted (using Planck's equation) to the $P A R$ flux to report the efficacy in micromoles/joule. Precision volt and current meters are used to simultaneously measure volts and amps and calculate power consumption. Detailed procedures for measuring input and output parameters are given in IESNA (2008a).

As an alternative to integrating sphere measurements, flat-plane integration can be used to quantify efficacy. This uses some of the same techniques as mentioned above but can be more labor intensive. Flatplane measurements must be made in specialized dark rooms (i.e., without any reflections from room surfaces). The lamp is typically mounted in a stationary position, and the sensor is moved to cover a series of measurement locations along a specific measurement plane. When the dark room is relatively small, measurements can be extrapolated (e.g., using curve-fitting techniques) to simulate a larger measurement surface (Nelson and Bugbee, 2014). These measurements can also be used to determine light-distribution patterns.

Light Distribution FROM ASYMMETRIC LAMP DESIGNS. As mentioned previously, the lamp described on the proposed label (Fig. 2) produced a uniform spectral distribution pattern irrespective of the direction the sensor was moved away (horizontally) from the center directly underneath the lamp. This may not be the case for all lamps and their reflectors, especially those with a rectangular design. In such cases, it may be necessary to include additional distribution curves on the proposed label that show distribution patterns in different directions. In that case, the lamp mounting orientation should be noted on the label. Typical curves used by the lighting industry include photometric distribution curves (side view) and contour plots (top-down views showing lines of constant light intensity).

LIGHT DISTRIBUTION AT DIFFERENT MOUNTING HEIGHTS. If light distribution is measured at different mounting heights, multiple mounting heights, or both, the resulting distribution curves can be added to the proposed label. If necessary and for clarity, multiple graphs can be included to show distribution curves at various mounting heights.
LAMP AGING. All lamps, including LEDs, produce less light as the number of operating hours increases. This will have an obvious impact on the performance characteristics of lamps. Growers are encouraged to periodically measure light output (preferably with a spectroradiometer) to evaluate the decline in performance. The lighting industry often uses a fixed depreciation to describe the useful life expectancy of a particular lamp type. For example, the term "L70" (IESNA, 2008b) is used to determine the number of operating hours after which the light output has dropped to $70 \%$ compared with that of a new lamp. Unless otherwise stated, lamp performance listed on the proposed label is for new lamps (measured after a customary burn-in period of $100 \mathrm{~h}$ ).

Chemical resistance (e.g., to SULFUR). Greenhouse growers often use sulfur vapor to control pests and fungal diseases, particularly thrips (Thrips sp.) and powdery mildew (order Erysiphales). Sulfur vapor has been known to accelerate the deterioration of plastic glazing materials and to cause corrosion of metals. In addition, other chemicals used to combat pests and diseases could have an impact on equipment (including lamps) used in greenhouses. Information about any of these impacts would be useful to growers, and if available, should be added to the label.

AdDitional information. If desirable, additional information can be added to the label. Examples include the presence of harmful chemicals, recycling or proper disposal instructions, protection level from humid or wet environments, and primary intended lamp uses. For example, some LED fixtures have been developed specifically to promote flowering of long-day plants and emit primarily red and far red radiation; such a lamp type may not be suitable to increase photosynthesis and plant growth.

AvaIlability OF THE PROPOSED LABEL. In contrast to the labels used for lamps for visible applications (which are often shown only on product packaging), we propose that manufacturers also post label information for lamps intended for plant growth applications on their product web sites.
Photobiological safety. Like plants grown inside, people working in greenhouses and controlled environments are exposed to the radiation emitted by electric lamps. Depending on intensity and duration, ultraviolet, blue, and IR radiation exposure can have a negative impact on a person's well-being and the impact can be different if that person has undergone certain medical procedures (e.g., cataract surgery). A publication discussing recommended practices (IESNA, 2015) addresses exposure limits. If adequate information is available to assess these exposure hazards to people working in plant production facilities, it should be made available and we recommend that it is included on the product label. For example, a manufacturer could recommend the use of protective eye-wear, a maximum exposure duration, or both.

\section{Literature cited}

Commission Internationale de l'Eclairage. 1931. Proceedings of the Eight Session. Cambridge Univ. Press, Cambridge, UK.

Gardner, G. and M.A. Graceffo. 1982. The use of a computerized spectroradiometer to predict phytochrome photoequilibria under polychromatic irradiation. Photochem. Photobiol. 36:349-354. doi: 10.1111/j.1751-1097.1982.tb04385.x.

Illuminating Engineering Society of North America (IESNA). 2004. LM-4604: Approved method for photometric testing of indoor luminaires using high intensity discharge or incandescent filament lamps (reaffirmed in 2012). Illuminating Eng. Soc. North Amer., New York, NY.

Illuminating Engineering Society of North America (IESNA). 2008a. LM-7908: Approved method for electrical and photometric measurements of solid-state lighting products. Illuminating Eng. Soc. North Amer., New York, NY.

Illuminating Engineering Society of North America (IESNA). 2008b. LM-8008: Approved method for measuring lumen maintenance of LED light sources. Illuminating Eng. Soc. North Amer., New York, NY.

Illuminating Engineering Society of North America (IESNA). 2015. ANSI/ IES RP-27.1-15: Recommended practices for photobiological safety for lamps and lamp systems - General requirements. Illuminating Eng. Soc. North Amer., New York, NY. 
McCree, K.J. 1972. The action spectrum, absorptance and quantum yield of photosynthesis in crop plants. Agr. Meteorol. 9:191-196. doi: 10.1016/0002-1771(71) 90022-7.

Mitchell, C.A., M.P. Dzakovich, C. Gomez, R. Lopez, J.F. Burr, R. Hernández, C. Kubota, C.J. Currey, Q. Meng, E.S. Runkle, C.M. Bourget, R.C. Morrow, and A.J. Both. 2015. Light-emitting diodes in horticulture. Hort. Rev. 43:1-87. doi: 10.1002/9781119107781.ch01.

Nelson, J.A. and B. Bugbee. 2014. Economic analysis of greenhouse lighting: Light emitting diodes vs. high intensity discharge fixtures. PLoS One 9(6):e99010 doi: 10.1371/journal.pone.0099010.

Nelson, J.A. and B. Bugbee. 2015. Analysis of environmental effects on leaf temperature under sunlight, high pressure sodium and light emitting diodes. PLoS One 10(10):e138930 doi: 10.1371/ journal.pone.0138930.

Sager, J.C., W.O. Smith, J.L. Edwards, and K.L. Cyr. 1988. Photosynthetic efficiency and phytochrome photoequilibria determination using spectral data. Trans. Amer. Soc. Agr. Eng. 31:1882-1889. doi: $10.13031 / 2013.30952$. 